\title{
Three-dimensional in vivo characterization of calcification in native valves and in Freestyle versus homograft aortic valves
}

Giovanni Melina, PhD, ${ }^{a}$ Paramate Horkaew, ${ }^{\text {b }}$ Mohamed Amrani, FRCS, ${ }^{a}$ Michael B. Rubens, FRCR, ${ }^{a}$ Magdi H. Yacoub, FRS, ${ }^{a}$ and Guang-Zhong Yang, $\mathrm{PhD}^{\mathrm{b}}$

Additional material is available online. $\bigcirc$
From the Academic Department of Cardiothoracic Surgery, National Heart and Lung Institute, Imperial College, University of London, ${ }^{\text {a }}$ and the Royal Society/Wolfson Foundation Medical Image Computing Laboratory, ${ }^{\mathrm{b}}$ London, United Kingdom.

Supported by the Royal Society/Wolfson Foundation, the British Heart Foundation, and in part by Medtronic Inc, Minneapolis, Minn.

G.M. and P.H. have contributed equally to this article.

Received for publication Dec 5, 2004; revisions received Jan 28, 2005; accepted for publication Feb 10, 2005.

Address for reprints: Professor Magdi Yacoub, The Magdi Yacoub Institute, Harefield Research Foundation, Hill End Rd, Harefield, Middlesex UB9 6JH, United Kingdom (E-mail: m.yacoub@imperial.ac.uk)

J Thorac Cardiovasc Surg 2005;130:41-7

$0022-5223 / \$ 30.00$

Copyright $\odot 2005$ by The American Association for Thoracic Surgery

doi:10.1016/j.jtcvs.2005.02.056
Objective: This article describes a novel interactive method for quantitative evaluation of calcium deposits in the aortic valve by means of electron beam tomography data fusion technique.

Methods: The technique relied on the use of hierarchic 3-dimensional free-form volume registration with fast global optimization between normally acquired and contrast-enhanced electron beam tomographic volume. A total of 66 contrastenhanced electron beam tomographic scans of the aortic root were performed in 27 patients, 10 with native aortic valve disease (group A) and 17 from a prospective randomized trial of aortic root replacement (group B, 9 Freestyle grafts [Medtronic, Inc, Minneapolis, Minn] and 8 homografts). To validate the in vivo electron beam tomographic measurements, 5 patients from group A underwent electron beam tomographic scans before the operation and then had their own valves, explanted at the time of surgery, analyzed for calcium quantification by ex vivo electron beam tomography.

Results: In group A, the mean ( \pm SE) calcification score was $6560 \pm 2388$, which correlated with peak gradients measured at echocardiography $(r=0.93, P=.02)$. In group B, the mean ( \pm SE) calcification score was $168 \pm 27$, showing a tendency toward a lower calcification for Freestyle valves than for homografts at 2 years after implantation $(P=.052)$. A mean variability of $6 \%$ was found between in vivo electron beam tomographic scores of calcification and those measured on valve specimens after explantation.

Conclusion: We describe a novel method to characterize the degree and location of calcification in both native valves and postoperative valve implants. The technique may be useful in the management of patients with aortic valve disease and has potential as a screening tool for high-risk patients to diagnose early valve calcification and possibly institute corrective measures.

$\mathrm{C}$ alcific aortic stenosis is the most common valve disease in elderly patients in Europe and the United States, and its incidence is rapidly rising. ${ }^{1,2}$ Factors implicated in atherosclerosis may contribute to valve calcification and therefore may be amenable to institution of therapeutic measures. ${ }^{3}$ In a recent prospective study of a series of symptom-free patients with severe aortic stenosis, the degree of calcification of the native aortic valve has been shown to be a determinant of prognosis and outcome. ${ }^{4}$ In addition, calcification of valve bioprostheses is thought to play a crucial role in their failure. ${ }^{5}$ Thus availability of a rapid and reliable technique for accurately quantifying the degree and pattern of calcification could play a major role in the management of both groups of patients and possibly serve as a screening tool for "high-risk" patients. Electron beam tomography (EBT) has been widely used to detect calcium in the coronary arteries ${ }^{6,7}$ and 
the aortic wall. ${ }^{8,9} \mathrm{~A}$ recent investigation ${ }^{10}$ with a computed tomographic (CT) technique demonstrated the pattern of calcification and graded the amount of calcium of the aortic valve, but that study was undertaken in patients with advanced calcific aortic stenosis and has not been validated. Similarly, Shavelle and associates ${ }^{11}$ used EBT to retrospectively quantify aortic valve calcification in patients who underwent EBT scanning to evaluate coronary artery calcium. They also demonstrated a threshold amount of EBT aortic valve calcification above which the probability of aortic stenosis is high. ${ }^{11}$ In addition, the group from Mayo Clinic have shown the prognostic value of EBT in detecting and quantifying calcium in the aortic valve. ${ }^{12}$ However, routine CT techniques are not able to localize the aortic valve leaflets unless they are heavily calcified because of the movement of the leaflets and aortic ring during different phases of the cardiac cycle. ${ }^{13}$ To address this, we have previously described a method that uses contrast enhancement EBT to localize the valve cusps on one occasion, which enables repeated scans after that. ${ }^{14}$ In addition, we have recently validated the EBT technique with both experimental $^{15}$ and human explanted bioprostheses. ${ }^{16}$ In this study, we aimed to refine and extend our previous method ${ }^{14}$ to quantify and localize calcium deposits with a rapid 3dimensional (3D) image registration technique in both native and postoperative bioprosthetic aortic valve leaflets.

\section{Methods}

\section{Patient Population and EBT Image Acquisition}

A total of 27 patients (age range 45-77 years) were investigated for EBT detection and quantification of calcium deposits in the aortic valve leaflets. This included 10 patients (group A) with aortic valve disease (stenosis in 6 and regurgitation in 4) and 17 postoperative patients (group B) from a prospective randomized trial of Medtronic Freestyle (Medtronic, Inc, Minneapolis, Minn) versus homograft root replacement ${ }^{9}$ at the Royal Brompton and Harefield National Health Service Trust (London, UK). In group B, 9 patients received Freestyle bioprostheses, and 8 received homograft valves. The local ethics committee approved the study protocol, and all participating patients gave written, informed consent to the investigation. EBT scans of the aortic root were performed before the operation in group A and at 6-monthly intervals after aortic root replacement in group B. The operative technique has been previously described. ${ }^{9}$ The clinical and demographic characteristics of the study population are listed in Table E1. In addition, a subset of 5 patients from group A undergoing aortic valve replacement had their diseased aortic valves carefully explanted at the time of surgery in an attempt to include all cusp calcification in one intact, excised specimen. The valve specimens so obtained were subsequently analyzed by EBT for calcium quantification, and the calcium scores thus measured were compared with those previously determined in vivo before the operation.

Before their scans, all patients were investigated regarding any known allergy, even though they had previously undergone cardiac catheterization with a larger amount of contrast medium as a routine investigation for their coronary arteries and possibly aortic valve pathology. Renal function was routinely checked for each patient.

For the in vivo EBT, a set of 20 to 25 transverse tomograms of 3-mm thickness was obtained through the aortic root with the subject breath holding. Acquisitions were made at $80 \%$ of the RR interval. The scan was then repeated during intravenous injection of a contrast medium. Omnipaque 240 (Nycomed Imaging AS, Oslo, Norway) was injected into a vein in the antecubital fossa at a rate of $3 \mathrm{~mL} / \mathrm{s}$, and scans were acquired at 30 seconds after the beginning of the injection. The explanted native aortic valves were analyzed in accordance with our previous studies. ${ }^{15,16}$

\section{Echocardiographic Measurements}

Transthoracic echocardiograms were performed at same time intervals as the EBT investigations by means of a Hewlett Packard Sonos (Hewlett-Packard Ltd, Bracknell, UK) machine. Echocardiographic Doppler imaging was used to analyze transvalvular and subvalvular flows. Peak transvalvular and subvalvular flow velocities were recorded, and gradients were derived with the modified Bernoulli equation: $\Delta \mathrm{P}=4 \mathrm{v}^{2}$. Mean gradients were calculated from the flow profile.

\section{EBT 3D Serial Image Registration}

The examinations were based on a sequential back-to-back scan. To circumvent the geometric inconsistencies introduced by the respiratory movements of the base of the heart during the cardiac cycle, we adapted a previously developed rapid intensity-based multiresolution image registration scheme. ${ }^{17}$ As extended from the previous study, ${ }^{17}$ a free-form image registration based on 3D B-spline transformation model was adopted to correct the respiration-induced cardiac deformation. In this study, intensity based cross-correlation coefficient was used as a measure of the degree to which the two images were aligned. The optimal B-spline parameters to maximize this similarity measure were determined with a multihierarchical quasi-Newton optimization strategy. ${ }^{17}$ The definition of the crosscorrelation coefficient and a detailed optimization strategy for image registration are described in Appendix E1.

\section{Interactive Volume Visualization and Calcium Scoring}

For interactive volume visualization of the general trends of valve calcification with time, a software-accelerated 3D interactive rendering technique called the "bubble model" with shear-warp factorization was used. The normally scanned volume was segmented with calcium-level thresholding (130 Hounsfield units) with isosurface rendering.

For quantitative analysis, interactive calcium quantification was conducted with CMRTools (Imperial College, London, UK) according to the Agatston scoring scheme. ${ }^{6,9}$ Two uninformed, independent observers (G.M and P.H.) were asked to interactively identify the location of the calcified leaflets and define regions of interest on the enhanced images showing the exact location of the valve leaflets. These regions of interest were then automatically overlaid on the corresponding unenhanced ones for calculation of the calcium score. A calcium score was then calculated for each valve by multiplying the calcified lesion area in square millimeters by a density score determined from the peak CT number. For each observer, the process was repeated twice. Both interobserver and intraobserver variabilities were calculated. In addition, on the basis 


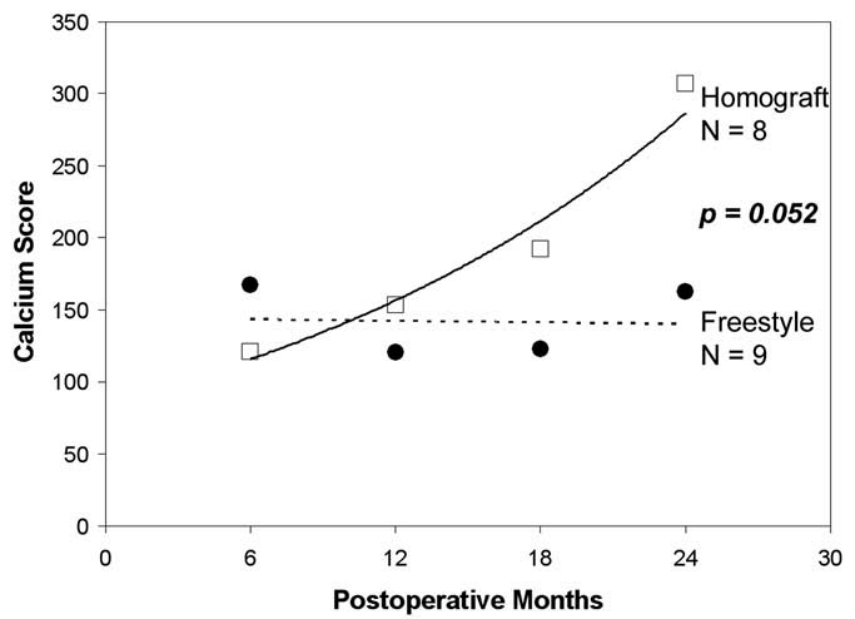

Figure 1. Calcification trend in patients who received bioprosthetic valves (homograft or Medtronic Freestyle).

of the free-form registration results, each aortic valve was normalized as a circular disk with sectors labeled as right, left, and noncoronary leaflets, respectively. For each subject, calcified lesions were spatially mapped onto the disk according to their actual locations on the leaflets. The use of this normalizing scheme not only facilitates intrasubject follow-up assessment but also simplifies localization comparisons. Calcium within aortic sinuses, aortic wall, or both was carefully excluded from the analysis.

\section{Statistical Analysis}

Continuous variables were analyzed by means of the Student $t$ test for unpaired data as appropriate. A 2-way analysis of variance was used to assess serial changes in continuous variables. Correlations between the calcification scores and peak transvalvular gradients were determined by means of Pearson product-moment correlation analysis.

\section{Results}

\section{Image Registration}

Figure E1 shows a single image slice through the 3D volume of a patient, illustrating both the conventional (Figure E1, A) and contrast-enhanced (Figure E1, B) EBT images. In Figure E1, $A$, the calcified region, in this case mainly on the aortic wall, is well defined. Because of similar X-ray attenuation in blood and the aortic cusps, the valvular region is not clearly visible. The injection of the contrast agent (Figure E1, B) enhances the attenuation difference and makes the aortic leaflets clearly delineated. Because of the inconsistencies between different breath-holds and discrepancies in patient alignment between serial examinations, Figure E1, $C$, demonstrates the misregistration artifact between the contrast-enhanced 3D volume and one of the serial scans. A composite color scheme of green and magenta is used. When two images are in alignment, the overlay image should generally be in gray.

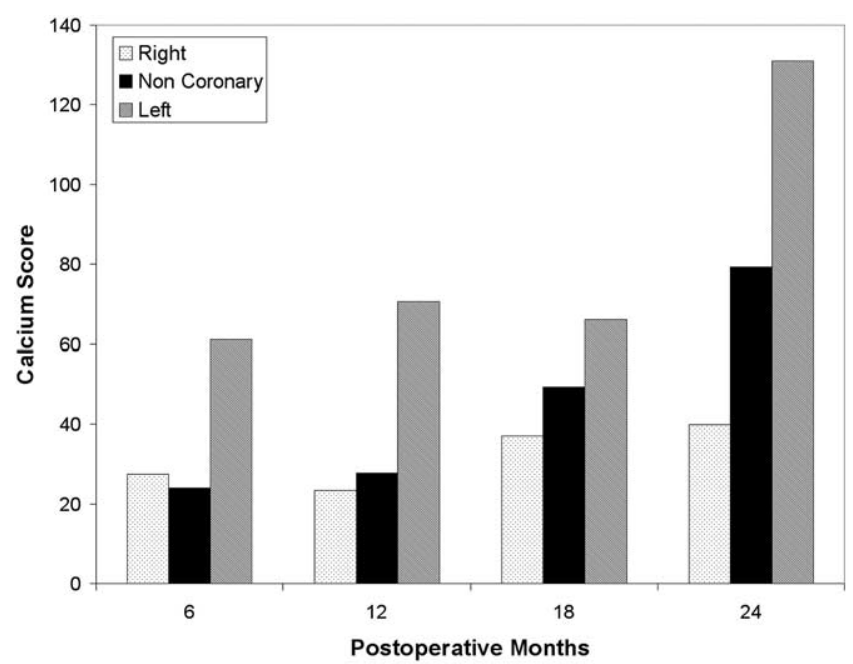

Figure 2. Calcium contents of left, right, and noncoronary cusps for all patients who underwent aortic valve replacement.

Figure E2 shows examples of color overlay of the normal and contrast-enhanced images before (Figure E2, $A$ ) and after (Figure E2, $B$ ) registration at three consecutive slice locations. It is evident that calcium deposits can be clearly identified around the aortic cusps after registration.

\section{Calcium Quantification}

The native dysfunctional aortic valves in group A had calcium scores from 235 to 16,332. The interobserver variability was $4 \%$, and the intraobserver variabilities were $2.2 \%$ and $2.5 \%$. Mean ( \pm SE) calcium scores were 10,586 \pm 3001 (mean $\pm \mathrm{SE}$ gradient $78 \pm 3 \mathrm{~mm} \mathrm{Hg}$ ) for patients with aortic stenosis and $523 \pm 177$ for patients with aortic regurgitation (mean $\pm \mathrm{SE}$ gradient $21 \pm 1 \mathrm{~mm} \mathrm{Hg}$ ).

The quantitative results for the 17 patients in group $\mathrm{B}$ are provided in Figure 1. There was a trend for calcification to increase with time in the homografts but not in the Freestyle valves. For the total 122 volume pairs analyzed, the interobserver variability was $9.2 \%$, and intraobserver variabilities were $18 \%$ and $13.5 \%$.

The calcium scores measured before the operation in the subset of 5 patients from group A varied from 870 to 16,332. The calcium quantification made on their own valves explanted during the operation showed scores varying from 771 to 15,239 , which gave a mean variability of $6 \%$ relative to those previously calculated in vivo.

\section{Calcium Localization}

Figure 2 shows the calcium content for each cusp (right, left, and noncoronary) with time in all the patients from group B. Interestingly, the left coronary leaflet showed a higher degree of calcification than did the right and noncoronary leaflets at each time point. 


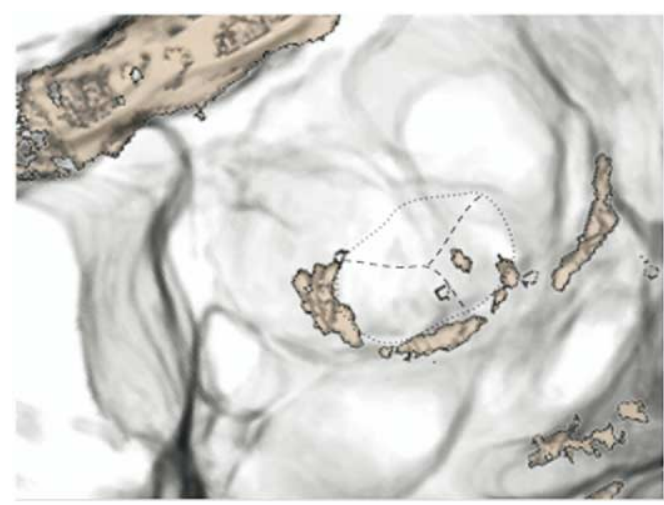

A

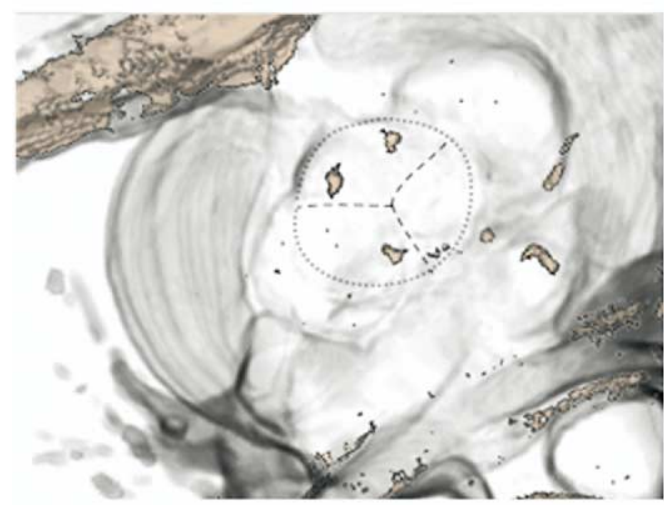

B

6 months

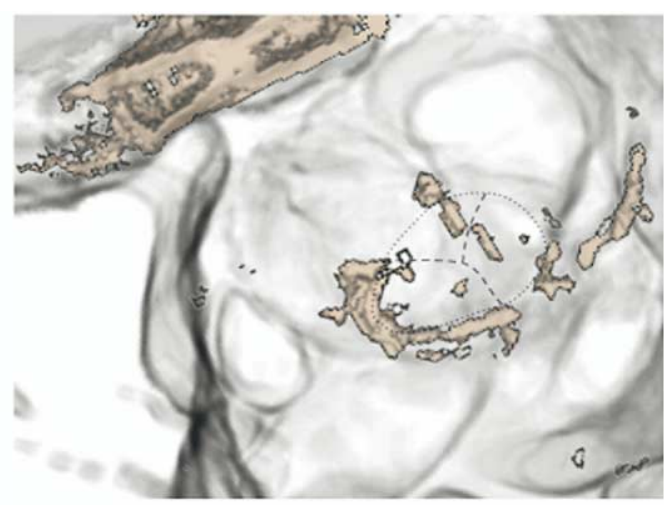

24 months

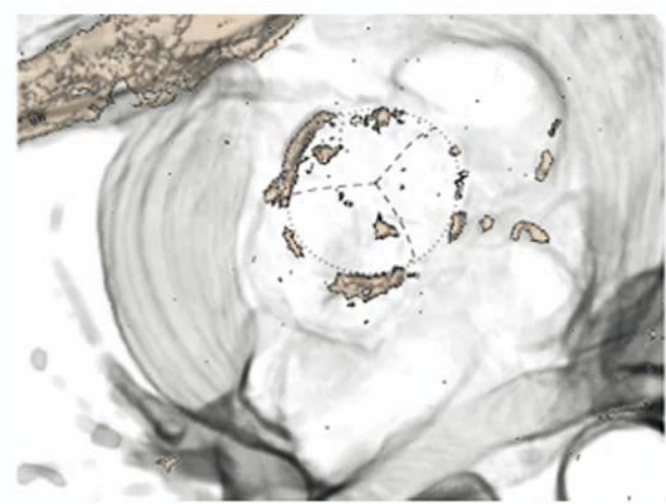

24 months

Figure 3. 3D rendering of overlaid volumes showing calcification after operation in homograft (A) and Medtronic Freestyle valve (B).

As shown in two samples in Figure 3, calcification was localized mainly at the base of the leaflets in the area of the native aortic annulus, corresponding to the proximal suture line in the group of patients who underwent aortic root replacement. In the homograft group, spots of calcification were widely detected in the belly of the leaflets at the latest EBT scan, particularly affecting the base (Figure E3, A). In the Freestyle group, minor degrees of calcification, which did not increase with time, remained localized at the suture line, with much smaller areas of calcification than in the homograft group in the belly of the cusp (Figure E3, B).

The native valves showed a higher degree of calcification of the three cusps (Figure E3,C). In this group, extensive calcification was found at the base of the leaflets, the belly, including the coaptation surface and at the three commissures (Figure E3, $C$ ).

\section{Relation to Hemodynamic Data}

In group $\mathrm{A}(\mathrm{n}=10)$, the mean peak transvalvular gradient measured at echocardiography was $54 \mathrm{~mm} \mathrm{Hg}$, and it cor- related well with the scores of calcification $(r=0.94, P=$ .02 ), with the highest calcification score to cause significant stenosis being 16,332 . No patient in group $B(n=17)$ showed pathologic gradients across the aortic valve. The mean gradients were always less than $3 \mathrm{~mm} \mathrm{Hg}$, and the echocardiographic investigations demonstrated a good leaflet excursion during the cardiac cycle at each interval. The highest calcification score in group B was 821 .

\section{Discussion}

This study describes a novel, rapid, 3D method of serial assessment of the amount and localization of calcium deposits in both native and bioprosthetic aortic valves. The technique requires a single contrast-enhanced baseline 3D tomographic volume for aligning subsequent conventional EBT scans that follow the development of calcified regions. The preliminary results from 27 patients demonstrate the technique's potential clinical use for calcium quantification in both native and bioprosthetic aortic valves. In addition, 
we have validated the use of this method for quantifying in vivo aortic valve calcification in human beings.

The aortic valve performs sophisticated functions, which are dependent on the dynamic behaviors of its components. ${ }^{18}$ Calcific stenosis is a common failure mode for both native human aortic valves and the biologic aortic bioprostheses, but in neither case are the etiology and mechanism of progression well understood. In native valves, calcification is an active process resembling atherosclerosis. ${ }^{3,19}$ The calcification process has some similarities in glutaraldehydefixed bioprosthetic valves, but it does not involve living interstitial cells because glutaraldehyde is cytotoxic. In addition, free aldehyde within the tissue matrix has also been thought to be an indicator for calcification. ${ }^{20}$ The biomechanical environment within which the bioprosthetic valve is placed determines the underlying trend that the structure is altered both morphologically and functionally. Existing research in the area underscores the complexity of the bioprosthetic valve calcification process and highlights the need for combined biochemical, genetic, biomechanical, histologic, and accurate in vivo imaging studies. The method described in this article could help in that regard.

EBT has proved to be highly sensitive for calcium measurements in the coronary arteries, ${ }^{21}$ and it has the advantage of being noninvasive, allowing a rapid acquisition of the images. By using EBT, we demonstrated the pattern of calcification of the aortic root wall after aortic root replacement with homografts or Medtronic Freestyle porcine xenografts. ${ }^{9}$ The technique was then improved for the visualization of the aortic valve leaflets. ${ }^{14}$ In two different in vitro experiments, we then validated our EBT method for detection and quantification of aortic valve leaflets. ${ }^{15,16} \mathrm{We}$ found high correlations between the EBT scores and of biochemically measured calcium content with experimental valves ${ }^{15}$ or failed biologic prostheses explanted from patients. ${ }^{16}$ The validation of the technique has been extended in this study, in which there was only a $6 \%$ mean variability between the EBT measures in vivo and the quantification of calcium on the aortic valve samples after explantation from the patients during the operation for aortic valve replacement. This variability could have been due, at least in part, to inability to excise the valve intact with all the calcium. The quantification of calcium was further improved in this investigation. We have used a fast, multiresolution 3D registration scheme based on the piecewise linear jointhistogram relationship between normally acquired and contrast-enhanced volumes. This simplifies the treatment of the optimization process, which involves a large number of free variables, and permits the use of Broyden-FletcherGoldfarb-Shanno (BFGS) algorithm, which is an extension to the Newton method and has a quadratic convergence property (Appendix E1). It is worth noting that the chosen similarity function is justified by the use of blood pools as registration landmarks. The local joint histogram of intensities within the blood pool has been shown to have nearly linear correlation between the standard and contrast-enhanced CT volumes. The abundance of the blood signal at the imaging volume therefore has a strong influence toward the correct alignment of both volumes. This patient study indicates the practical value of the developed method.

Some recent studies have suggested the use of EBT to detect and quantify calcification of the native aortic valve. $^{3,22}$ These studies, however, tended to use scans performed primarily to assess coronary artery calcification ${ }^{3}$ or were done in patients undergoing catheterization before aortic valve replacement or who were found to have at least mild aortic stenosis at echocardiography ${ }^{22}$ and therefore did not take into account the movement of the aortic root, the position of the aortic cusps, and the different positioning of the patients on the scanner. These difficulties can be circumvented by localizing the aortic cusps with contrast enhancement on one occasion. ${ }^{14}$ Shavelle and associates ${ }^{11}$ showed in a retrospective study that patients with EBT aortic valve calcification scores of at least 150 should be referred for echocardiography, because these are more likely to have significant aortic stenosis. ${ }^{11}$ In our series of bioprosthetic valves (both homografts and Freestyle valves), 8 patients had a calcification score of at least 150 at different time points, but in none were peak gradients higher than $7 \mathrm{~mm} \mathrm{Hg}$. This rule of thumb, however could be true for the native valves, where the lowest score was 235 and the agreement with the echocardiographic gradients across the aortic valve was high. We think this demonstrates the importance of the accurate determination of the aortic valve leaflets and the quantification of calcium by one method.

Calcification is thought to be a major determinant of hemodynamic function and durability of biologic devices, which leads to stenosis or regurgitation after implantation. Localized calcification can alter the physical properties of the valve and encourage tearing in bioprostheses.

In an attempt to enhance the performance of bioprostheses, different anticalcification treatments are being used. ${ }^{23,24}$ Although these techniques have met with success in animal models, the efficacy in human beings has not been investigated. A quantitative evaluation of the impact of such treatments is therefore needed, and the technique described in this article provides a powerful tool to achieve this goal. In addition, the technique allows evaluation of the rate of progression of calcification in both the native and bioprosthetic aortic valves and screening of people at risk such as middle-aged individuals, atherosclerotic patients, patients undergoing treatment of renal failure, postmenopausal women, and patients affected by familial hypercholesterolemia, which could be of prognostic importance. In addition, the technique described in this article provides for the first 
time data relating to the in vivo 3D localization of the calcium deposits in the valve. This provides new additional data relating to the pattern of calcification in different types of valves and could help in the understanding of the pathogenesis of calcification and possibly of its prognosis.

Until recently, the most accurate methods of quantifying bioprosthetic valve calcification consisted of in vitro analyses such as histologic assessments, radiographic and atomic absorption spectroscopy, and dual energy x-ray absorptiometry, ${ }^{25}$ which did not allow sequential examinations in the same patient. This study, which was conducted in patients, confirmed that calcification of the bioprosthetic aortic leaflets occurs as early as 6 months after implantation for both homografts and Freestyle prostheses. There was a trend for the calcification to progressively increase in the homograft group up to 2 years after the operation. The trend was different in the Freestyle group, where calcification tended to remain constantly low throughout all the investigation period. The amounts of calcium documented in the bioprosthetic valves were much lower than those observed in the diseased native valves. This could be explained by the fact that the homograft and Freestyle valves studied were well functioning and had no echocardiographic evidence of stenosis. However, the progressive accumulation of calcium in the homografts is a cause for concern and warrants further investigation. One of the limitations of this study is that it does not provide information about the dynamics of the valve leaflets because of the intrinsic constraint of the imaging modality itself. Recent developments of objecttracked magnetic resonance imaging ${ }^{26}$ and 3D ultrasonography ${ }^{27}$ are likely to play an important role in future investigations in linking mechanical properties to the progression of valve calcification. Another limitation is that the use of a contrast medium may be a potential risk for patients with renal failure and is also a contraindication for allergic patients.

In conclusion, the technique of in vivo 3D characterization of aortic valve calcification described in this article appears to be a sensitive, reliable method of measuring the amount and localization of calcification in both native and bioprosthetic valves. Sequential use of this technique could be valuable in understanding the pathogenesis of valve calcification and in the management of patients with aortic valve disease or bioprosthetic valve replacement.

\section{References}

1. Otto CM. Aortic stenosis. Listen to the patient, look at the valve. $N$ Engl J Med. 2000;343:652-4.

2. Otto CM, Lind BK, Kitzman DW, Gersh BJ, Siscovick DS. Association of aortic valve sclerosis with cardiovascular mortality and morbidity in the elderly. $N$ Engl J Med. 1999;341:142-7.

3. Pohle K, Maffert R, Ropers D, Moshage W, Stilianakis N, Daniel WG, et al. Progression of aortic valve calcification. Association with coronary atherosclerosis and cardiovascular risk factors. Circulation. 2001; 104:1927-32.
4. Rosenhek R, Binder T, Porenta G, Lang I, Christ G, Schemper M, et al. Predictors of outcome in severe, asymptomatic aortic stenosis. N Engl J Med. 2000;343:611-7.

5. Schoen FJ, Levy RJ, Nelson AC, Bernhard WF, Nashef A, Hawley M. Onset and progression of experimental bioprosthetic heart valve calcification. Lab Invest. 1985;52:523-32.

6. Agatston AS, Janowitz WR, Hildner FJ, Zusmer NR, Viamonte M Jr, Detrano R. Quantification of coronary artery calcium using ultrafast computed tomography. J Am Coll Cardiol. 1990;15:827-32.

7. Ludman PF, Lazem F, Barbir M, Yacoub M. Incidence and clinical relevance of coronary calcification detected by electron beam computed tomography in heart transplants recipients. Eur Heart J. 1999; 20:303-8.

8. Hoeg JM, Feuerstein IM, Tucker EE. Detection and quantitation of calcific atherosclerosis by ultrafast computed tomography in children and young adults with homozygous familial hypercholesterolemia. Arterioscler Thromb. 1994;14:1066-74.

9. Melina G, Rubens MB, Birks EJ, Bizzarri F, Khaghani A, Yacoub MH. A quantitative study of calcium deposition in the aortic wall following Medtronic Freestyle compared to homograft aortic root replacement. A prospective randomised trial. J Heart Valve Dis. 2000; 9:97-103.

10. Willmann JK, Weishaupt D, Lachat M, Kobza R, Roos JE, Seifert B, et al. Electrocardiographically gated multi-detector row CT for assessment of valvular morphology and calcification in aortic stenosis. Radiology. 2002;225:120-8.

11. Shavelle DM, Budoff MJ, Buljubasic N, Wu AH, Takasu J, Rosales J, et al. Usefulness of aortic valve calcium scores by electron beam computed tomography as a marker for aortic stenosis. Am J Cardiol. 2003;92:349-53.

12. Messika-Zeitoun D, Aubry MC, Detaint D, Bielak LF, Peyser PA, Sheedy PF, et al. Evaluation and clinical implications of aortic valve calcification measured by electron-beam computed tomography. Circulation. 2004;110:356-62.

13. Melina G, Rubens MB, Yacoub MH. Statins, electron-beam CT and aortic valve calcification. Lancet. 2002;360:258.

14. Melina G, Rubens MB, Amrani M, Khaghani A, Yacoub MH. Electron beam tomography for cusp calcification in homograft versus Freestyle xenografts. Ann Thorac Surg. 2001;71(5 Suppl):S368-70.

15. Melina G, Scott MJ, Cunanan CM, Rubens MB, Yacoub MH. Validation of the electron beam tomography method for valve cusp calcification. J Heart Valve Dis. 2002;11:402-8.

16. Melina G, Horkaew P, Yang GZ, Toomes C, Myers D, Yacoub MH. Validation of electron beam tomography quantification of valve calcification using human biological valve samples [abstract]. Circulation. 2003;107:e131.

17. Veeser S, Dunn MJ, Yang GZ. Multiresolution image registration for two-dimensional gel electrophoresis. Proteomics. 2001;1:856-70.

18. Yacoub MH, Kilner PJ, Birks EJ, Misfeld M. The aortic outflow and root: a tale of dynamism and crosstalk. Ann Thorac Surg. 1999;68(3 Suppl):S37-43.

19. Mohler ER 3rd, Gannon F, Reynolds C, Zimmerman R, Keane MG, Kaplan FS. Bone formation and inflammation in cardiac valves. Circulation. 2001;103:1522-8.

20. Pettenazzo E, Deiwick M, Thiene G, Molin G, Glasmacher B, Martignago $\mathrm{F}$, et al. Dynamic calcification of bioprosthetic porcine valves: evidence of apatite crystallization. J Thorac Cardiovasc Surg. 2001; 121:500-9.

21. Budoff MJ, Georgiou D, Brody A, Agatston AS, Kennedy J, Wolfkiel $\mathrm{C}$, et al. Ultrafast computed tomography as a diagnostic modality in the detection of coronary artery disease: a multicenter study. Circulation. 1996;93:898-904.

22. Kizer JR, Gefter WB, deLemos AS, Scoll BJ, Wolfe ML, Mohler ER 3rd. Electron beam computed tomography for the quantification of aortic valvular calcification. J Heart Valve Dis. 2001;10:361-6.

23. Gott JP, Pan-Chih, Dorsey LM, Jay JL, Jett GK, Schoen FJ, et al. Calcification of porcine valves: a successful new method of antimineralization. Ann Thorac Surg. 1992;53:207-16. 
24. Vyavahare N, Hirsch D, Lerner E, Baskin JZ, Schoen FJ, Bianco R, et al. Prevention of bioprosthetic heart valve calcification by ethanol preincubation. Efficacy and mechanisms. Circulation. 1997; 95:47988.

25. Boughner DR, Thornton M, Dunmore-Buyze J, Holdsworth DW. The radiographic quantification of aortic valve calcification: implications for assessing bioprosthetic valve calcification in vitro. Physiol Meas. 2000;21:409-16.
26. Ringgaard S, Botnar RM, Djurhuus C, Stodkilde-Jorgensen H, Hasenkam JM, Boesiger P, et al. High-resolution assessment of velocity fields and shear stresses distal to prosthetic heart valves using highfield magnetic resonance imaging. J Heart Valve Dis. 1999;8:96-103.

27. Mannaerts H, Li Y, Kamp O, Valocik G, Hrudova J, Ripa S, et al. Quantitative assessment of mechanical prosthetic valve area by 3dimensional transesophageal echocardiography. J Am Soc Echocardiogr. 2001;14:723-31. 


\section{Appendix E1. 3D Multiresolution Registration}

Extended from our previous study of fast 2-dimensional image registration, the registration procedure was carried out by using the cross-correlation coefficients. It was chosen as the similarity measure because its mathematical formulation can be exploited to speed up the optimization process, as discussed by Veeser and colleagues. ${ }^{17}$ That is:

$\operatorname{sim}(I 1, I 2)$

$$
=\left|\frac{\sum_{\vec{x} \in \Omega}(I 1(\vec{x})-E\{I 1\})(I 2(\vec{x})-E\{I 2\})}{\sqrt{\sum_{\vec{x} \in \Omega}(I 1(\vec{x})-E\{I 1\})^{2}} \sqrt{\sum_{\vec{x} \in \Omega}(I 2(\vec{x})-E\{I 2\})^{2}}}\right|
$$

where $\Omega$ is the overlapping voxels in the intersection region of the reference and the transformed volume, $E\{\cdot\}$ is an expecting function of the voxels intensity, and $I 1, I 2$ is the referent and target images to be registered.

A linear tensor product B-spline defined by equation 2 was subsequently used as the transformation matrix because it enables adaptive subdivision of the control grid for a multiresolution implementation; that is:

$$
T(\vec{p})=\sum_{i, j, k} \beta_{i j k}(u, v, w) \vec{c}_{i j k}
$$

where $\beta_{i j k}(u, v, w)$ is the coefficient applied to the point $\rightarrow p(u, v, w)$ $c_{i j k}$ is the coordinate of control points surrounding the point $\rightarrow p(u, v, w)$, and $T(\rightarrow p)$ is the transformation that map point $\rightarrow p(u, v, w)$ in target image to referent image.

A quasi-Newton optimization algorithm, the BFGS algorithm, was then used to find the optimum transformation parameter vectors. In contrast to the Newton method, the algorithm does not need explicit second order derivatives. Instead, it approximates these values by using information from previous iterations; that is:

$$
\begin{aligned}
x_{k+1} & =x_{k}-H_{k}^{-1} \nabla f\left(x_{k}\right), \\
s_{k} & =x_{k+1}-x_{k}, \quad y_{k}=\nabla f\left(x_{k+1}\right)-\nabla f\left(x_{k}\right), \\
H_{k+1} & =H_{k}+\frac{y_{k} y_{k}^{T}}{y_{k}^{T} s_{k}}-\frac{H_{k} s_{k} s_{k}^{T} H_{k}}{s_{k}^{T} H_{k} s_{k}}
\end{aligned}
$$

where $x_{k}$ is the parameter vector in search space at $k$ th iteration, $f\left(x_{k}\right)$ is the objective function, $\nabla f\left(x_{k}\right)$ is the vector of partial derivative of the function with respect to each parameter, and $H_{k}$ is the estimated Hessian matrix.

As indicated by equation 3, the BFGS algorithm, however, does require the evaluation of the first order derivatives of the objective function, which can be computationally expensive. Fortunately, with the structure of the similarity matrix, the partial derivatives of the similarity measure with respect to the control points can be expressed analytically; that is:

where,

$$
\nabla f(\vec{c})=\left[\frac{\partial f}{\partial c_{1}^{x_{0}}} \frac{\partial f}{\partial c_{1}^{x_{1}}} \frac{\partial f}{\partial c_{1}^{x_{2}}} \cdots \frac{\partial f}{\partial c_{n}^{x_{0}}} \frac{\partial f}{\partial c_{n}^{x_{1}}} \frac{\partial f}{\partial c_{n}^{x_{2}}}\right]^{T}
$$

$$
\begin{array}{r}
\left.\frac{\partial \operatorname{cov}\left(I_{1}(\vec{p}), I_{2}\left(T_{c}[\vec{p}]\right)\right)}{\partial c_{j}^{x_{i}}}\right] \cdot \sigma\left(I_{2}\left(T_{c}[\vec{p}]\right)\right) \\
-\operatorname{cov}\left(I_{1}(\vec{p}), I_{2}\left(T_{c}[\vec{p}]\right)\right) \cdot \\
\frac{\partial f}{\partial c_{j}^{x_{i}}}=\frac{\left[\frac{\partial \sigma\left(I_{2}\left(T_{c}[\vec{p}]\right)\right)}{\partial c_{j}^{x_{i}}}\right]}{\sigma\left(I_{1}\right) \sigma^{2}\left(I_{2}\left(T_{c}[\vec{p}]\right)\right)}
\end{array}
$$

and

$$
\begin{gathered}
\frac{\partial \operatorname{cov}\left(I_{1}(\vec{p}), I_{2}\left(T_{c}[\vec{p}]\right)\right)}{\partial c_{j}^{x_{i}}}=\operatorname{cov}\left(I_{1}(\vec{p}), \frac{\partial I_{2}\left(T_{c}[\vec{p}]\right)}{\partial c_{j}^{x_{i}}}\right) \\
\frac{\partial \sigma\left(I_{2}\left(T_{c}[\vec{p}]\right)\right)}{\partial c_{j}^{x_{i}}}=\frac{\operatorname{cov}\left(I_{2}(T[\vec{p}]), \frac{\partial I_{2}\left(T_{c}[\vec{p}]\right)}{\partial c_{j}^{x_{i}}}\right)}{\sigma\left(I_{2}(T[\vec{p}])\right)} \\
\begin{array}{ccc}
\frac{\partial I_{2}(T[\vec{p}])}{\partial c_{j}^{x_{i}}}= & {\left[\begin{array}{lll}
\frac{\partial I_{2}(T[\vec{p}])}{\partial x_{0}} & \frac{\partial I_{2}(T[\vec{p}])}{\partial x_{1}} & \frac{\partial I_{2}(T[\vec{p}])}{\partial x_{2}}
\end{array}\right]} \\
\cdot\left[\begin{array}{lll}
\frac{\partial T_{x_{0}}[\vec{p}]}{\partial c_{j}^{x_{i}}} & \frac{\partial T_{x_{1}}[\vec{p}]}{\partial c_{j}^{x_{i}}} & \frac{\partial T_{x_{2}}[\vec{p}]}{\partial c_{j}^{x_{i}}}
\end{array}\right]^{T}
\end{array}
\end{gathered}
$$

which leads to an efficient implementation of the optimization strategy. 


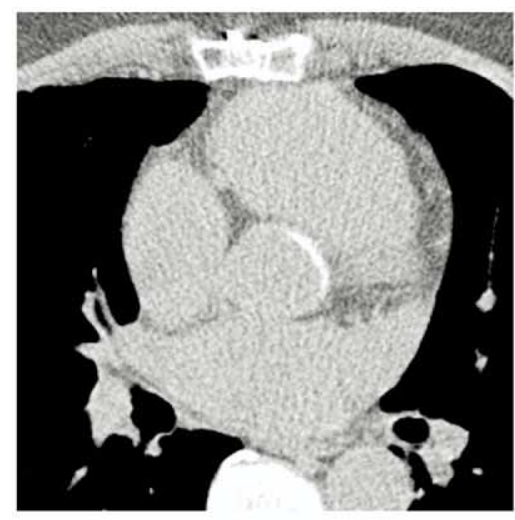

A

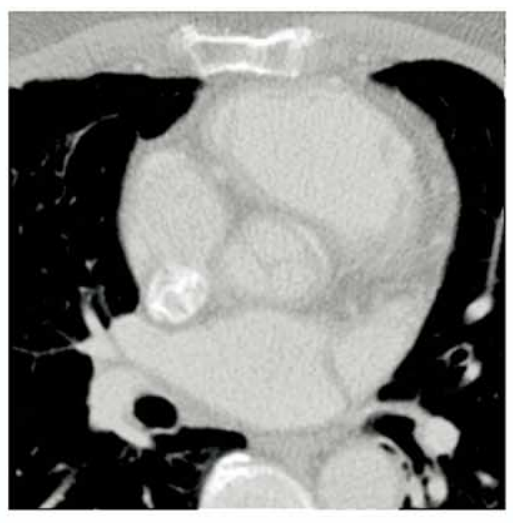

B

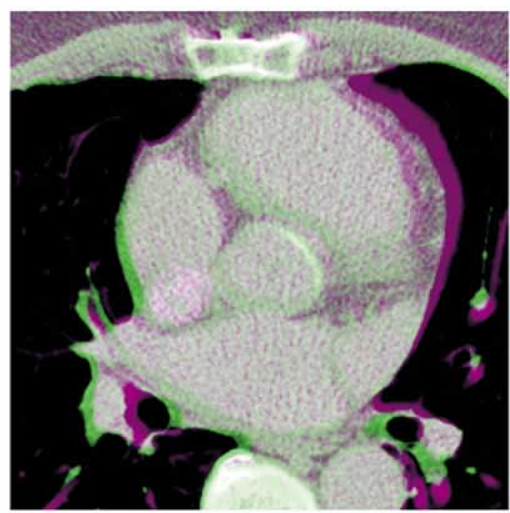

C

Figure E1. One slice of 3D volume before (A) and after (B) contrast enhancements and their superposition (C) with green-magenta color scheme, showing misregistration artifacts.

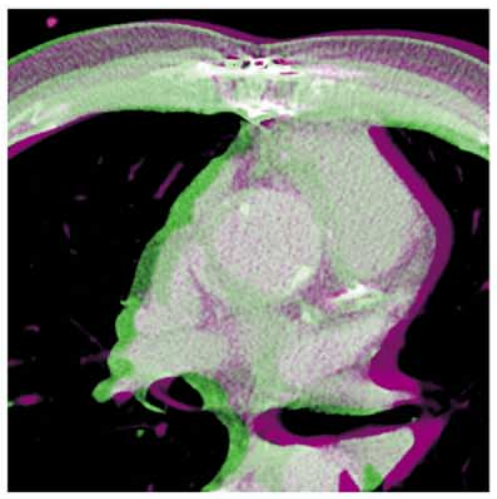

(A-1)

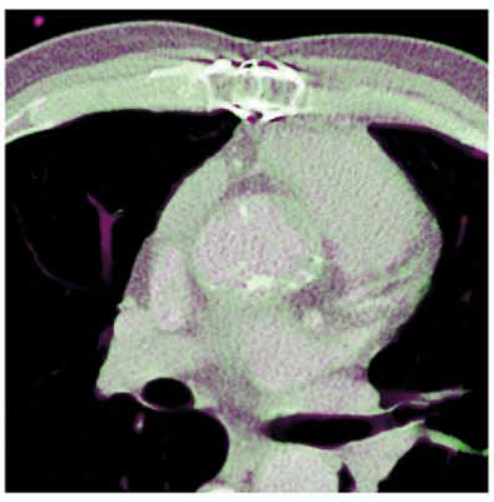

(B-1)

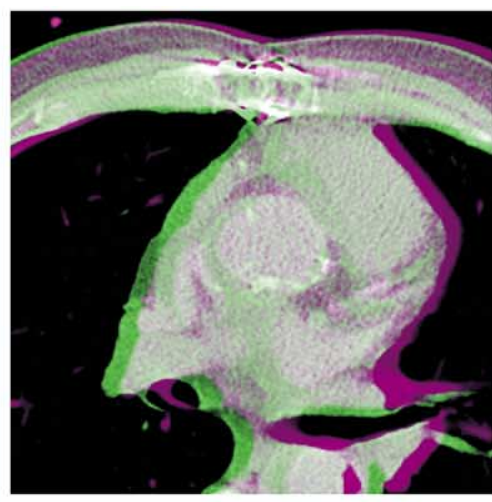

(A-2)

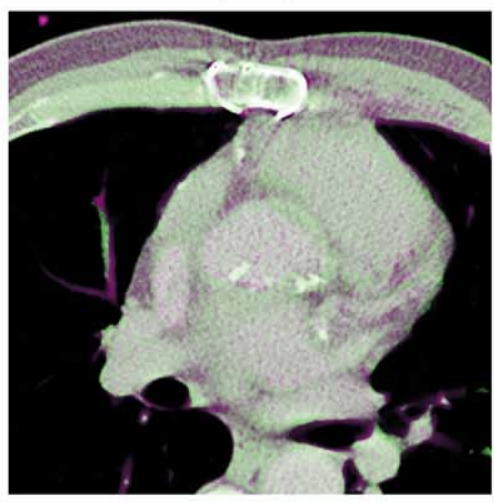

(B-2)

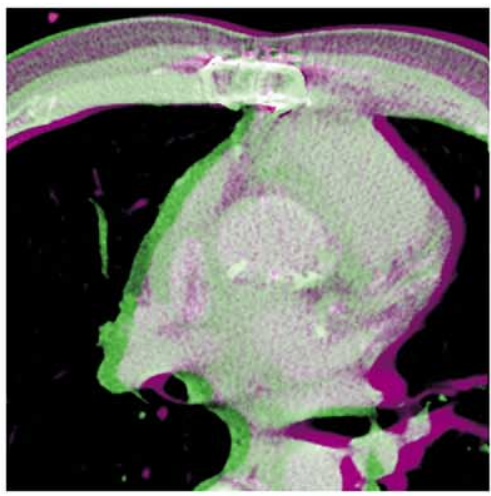

(A-3)

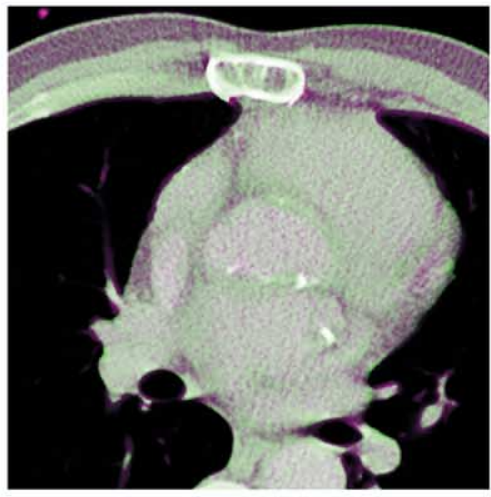

(B-3)

Figure E2. Color overlay of three consecutive slices before $(A, 1-3)$ and after $(B, 1-3)$ registration, showing influence of new method of correcting misregistration (magenta color). 

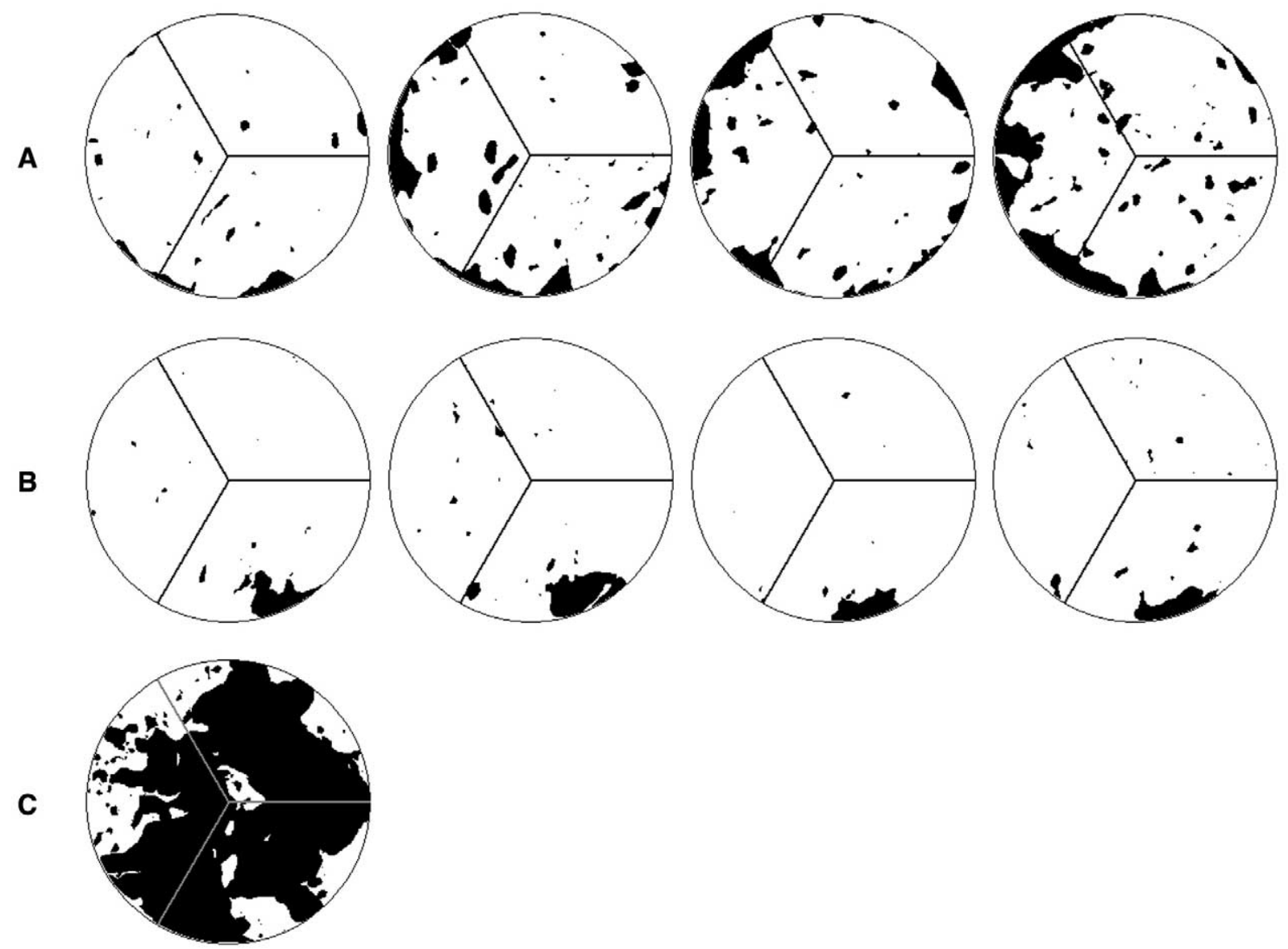

Figure E3. Normalized aortic valve showing localization of calcification in homograft group (A), Freestyle group (B), and native valve group (C).

\section{TABLE E1. Patient characteristics}

\begin{tabular}{lcc}
\hline & Group A & Group B \\
\hline No. of patients & 10 & 17 \\
Age (y) & & \\
$\quad$ Mean \pm SE & $62 \pm 2$ & $66 \pm 2$ \\
$\quad$ Range & $49-77$ & $45-75$ \\
Sex (male/female) & $8: 2$ & $12: 5$ \\
Smoking (\%) & 20 & 41 \\
Hypertension (\%) & 40 & 35 \\
Diabetes (\%) & 0 & 0 \\
Renal failure (\%) & 0 & 0 \\
Coronary artery disease (\%) & 20 & 29 \\
Transvalvular gradient & $54 \pm 3$ & $6 \pm 1$ \\
$\quad$ (mm Hg, mean \pm SE) & & \\
\hline
\end{tabular}

